\title{
Energy Consumption Monitoring System for Large Complexes
}

\author{
André Jorge, João Guerreiro, Pedro Pereira, João Martins, and Luís Gomes \\ UNINOVA - CTS \\ Universidade Nova de Lisboa - Faculdade de Ciências e Tecnologia \\ Monte da Caparica, Portugal \\ andrejorge77@gmail.com, joaocaguerreiro@gmail.com, \\ \{pmrp,jf.martins\}@fct.unl.pt, lugo@uninova.pt
}

\begin{abstract}
This paper describes the development of an open source system for monitoring and data acquisition of several energy analyzers. The developed system is based on a computer with Internet/Intranet connection by means of RS485 using Modbus RTU as communication protocol. The monitoring/metering system was developed for large building complexes and was validated in the Faculdade de Ciências e Tecnologia University campus. The system considers two distinct applications. The first one allows the user to verify, in real time, the energy consumption of any department in the complex, produce load diagrams, tables and print, email or save all available data. The second application keeps records of active/reactive energy consumption in order to verify the existence of some anomalous situation, and also monthly charge energy consumption to each corresponding department.
\end{abstract}

Keywords: Power Meter, Power Analyzer, Energy Efficient Buildings, Modbus Protocol.

\section{Introduction}

Environmental conservation has become a very important issue. The use of renewable energies has experienced a significant growth from the crisis of the oil in the 70's, in which renewable forms of energy started to be considered as a potentially alternative to the oil producing finite resources of the Earth [1]. Recent European Community Directives point to an energy consumption reduction, leading to an annual improvement in energy efficiency of around 6 per cent in 2012. Strict regulations, regarding power consumption and energy efficiency, have been set to preserve the environment. To fulfill energy conservation goals, it will be helpful if consumers could assess their energy load diagram so that they can plan/re-plan their energy consumption profile. Unstable global energy supplies, increasing load demands and increasing environment concerns are forcing energy producers and consumers to re-evaluate the energy usage paradigm. An energy monitoring system is an essential tool to help consumers understanding their energy usage profile and the associated environment impact and 
providing an instrument to re-shape their energy load diagram and, consequently, reduce its inherent costs. The developed system helps and instructs people to use their energy in a better way.

The efficient use of energy has direct consequences for the users, such as consumption and inherent costs reduction. Several strategies can be taken to fulfill these objectives: load shifting, automatic load control, energy storage, autonomous generation, and renewable energy usage. Nevertheless, in order to implement any of those strategies it is fundamental to accurately know the load diagram and load profile of each user. There are some commercial tools that can be used to monitor energy consumption and perform power management analysis, however when large complexes, with hundreds of metering points, are considered they become extremely expensive. In [2], Yeardy et al. present another tool for power consumption measurement, showing some results.

The main purpose of the present work is the development of a open source distributed metering system, with an innovative user interface and communication tool, that allows the analysis and study of energy load profile, applied to large complexes. The system was successfully installed in the campus of the Faculty of Science and Technology (Universidade Nova de Lisboa), Portugal. The campus comprises several buildings' departments occupying a total area of 30ha. The development system proposes a method to circumvent the limitations placed by the Modbus protocol [3][4] (as the typical series communication structure, improving the transmission rate), acquire energy consumption data, produce load diagrams for each user in the complex facility (allowing users to evaluate potential interventions in order to reduce consumption and minimize waste and load peaks), calculate the energy consumption costs associated with each department, maintain monthly costs accounting records, and provide detailed information on the validity of best rates solutions. The development of the system was based on three phases:

1. Development of the network communication tools;

2. Development of the real time analyses software package;

3. Development of the energy recording, taxing and analysis software package;

The last package is essential for organizing the energy cost distribution between all campus' departments, and help each one of them establishing solutions that should be adopted in order to solve the problem of excessive energy consumption [5].

\section{Contribution to Technological Innovation}

Current energy consumption is based on conventional data logging systems, which involves two major problems; (i) is the fact that retrieving the energy consumption data is not conventional, (ii) the cost of energy consumption monitoring systems is high. Smart-meters offers a possible solution, however they are often proprietary equipment with dedicated protocols. The solution presented here is an open-source energy monitoring system that skirts the above barriers by also being cheap and robust. 


\section{Modbus Protocol}

Modbus is an open protocol used in Industrial Automation Systems (IAS) as well as in Building Management Systems (BMS). The Modbus protocol was created in 1979 by Modicon, a company of Schneider Electric Group. In 2004 this group decided to offer Modbus as a public protocol and by that reason, the Modbus-IDA community appeared [6]. Since 1979 Modbus protocol rapidly became an industrial standard and has been implemented by hundreds of manufacturers in various applications and industrial segments. Its popularity has increased due to its simplicity of implementation and availability of free source code in the Internet. Modbus is an application-layer messaging protocol, positioned at level 7 of the OSI model and provides client/server communication between devices connected on different types of buses or networks.

The devices connected to a Modbus network interact through a master-slave scheme where only the master can start the conversation and the slave responds by sending the information requested by the master or performing the task set for him.

Serial Modbus connections can use two basic transmission modes, namely ASCII (American Standard Code for Information Interchange) or RTU (remote terminal unit). The transmission mode in serial communications defines the way the Modbus messages are encoded. Using Modbus/ASCII, the messages use plain ASCII format, while when using Modbus/RTU format a compressed format is used. This binary encoding makes the message unreadable by humans when monitoring, but have a severe impact in terms of reducing the size of each message, which allows for more data exchange in the same time frame.

\section{Network Hardware Description}

Fig. 1 presents proposed network topology, composed by the following main devices:

- $\quad$ PD8 (RS-485/Ethernet Converter)

- UPT210 Universal Power Transducer

- CT (50:5 current transformer)

The PD8 (RS-485/Ethernet Converter) converter allows transmitting data from/to master devices through an Ethernet data network, to/from devices equipped with an RS-485 interface. The converter is available in two versions concerning its operation mode: Real-Port network service and serial bridge with UDP protocol. In our particular case, the converter is operating with Real-Port Network service because a computer was needed to log the information provided by the UPT210. The converter used in this mode enables to co-operate only with one master computer at a time. In this case, for using a PD8 converter to serve Modbus protocol, a PC is needed to control break time intervals between received transmission characters.

UPT210 Universal Power Transducer is a digital meter able to measure the electrical parameters on three-phase systems. It provides accurate measurements even by distorted waveform. An LCD display provides the three-phase quantities. The 
working parameters can be easily set up by instrument keypad. UPT210 is a compact, cost effective meter operating both as a stand-alone device and as an integral part of a more extensive energy monitoring and management network. It replaces multiple analogue meters as well as single function meters such as voltmeters, ammeters, wattmeters, varmeters, frequency-meters, power factor-meters, energy-meters, etc.

The current transformer (CT) is used for measurement of electric currents. When current in a circuit is too high to directly apply to measuring instruments, a current transformer produces a reduced current accurately proportional to the current in the circuit (in this case, 50:5 CT), which can be conveniently connected to measuring and recording instruments. The current transformer also isolates the measuring instruments from what may be very high voltage in the primary circuit.

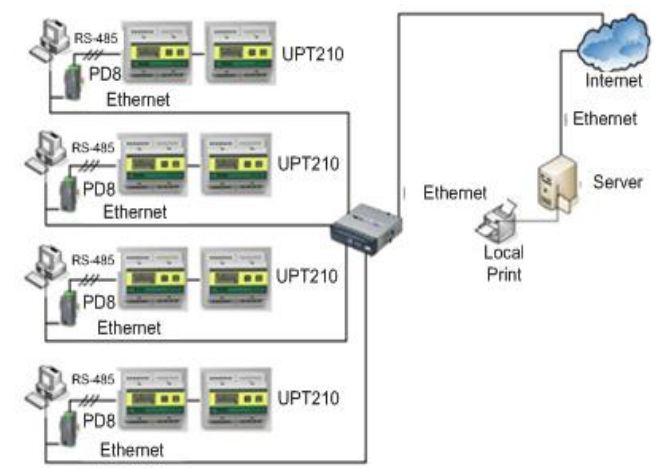

Fig. 1. Network topology

\section{Network Implementation Details}

In this section, we will step through each part of the network implementation, which is broken down in two main areas: Master implementation and Slave implementation.

The first one, Master implementation, is composed by a computer running a monitor program, having as main tasks to control break time intervals between received and transmitted message, gather information from all the transducers and analyze the collected data.

Using a computer as the system brain, allows to employ meters without local memory, which are cheaper compared with those with it, and from a single place can collect all the data, making unnecessary any human resources to do this job.

The second area, concerns all the physical devices in the neighbourhood of the place where power consumption is going to be measured. This includes several power transducers and a PD8 converter which allows the communication between the local network or internet and the devices equipped with RS-485 interface.

The system can be implemented either in a local network or using internet for remote access, as shown in Fig. 2. In this work, the system was tested in a local network. 


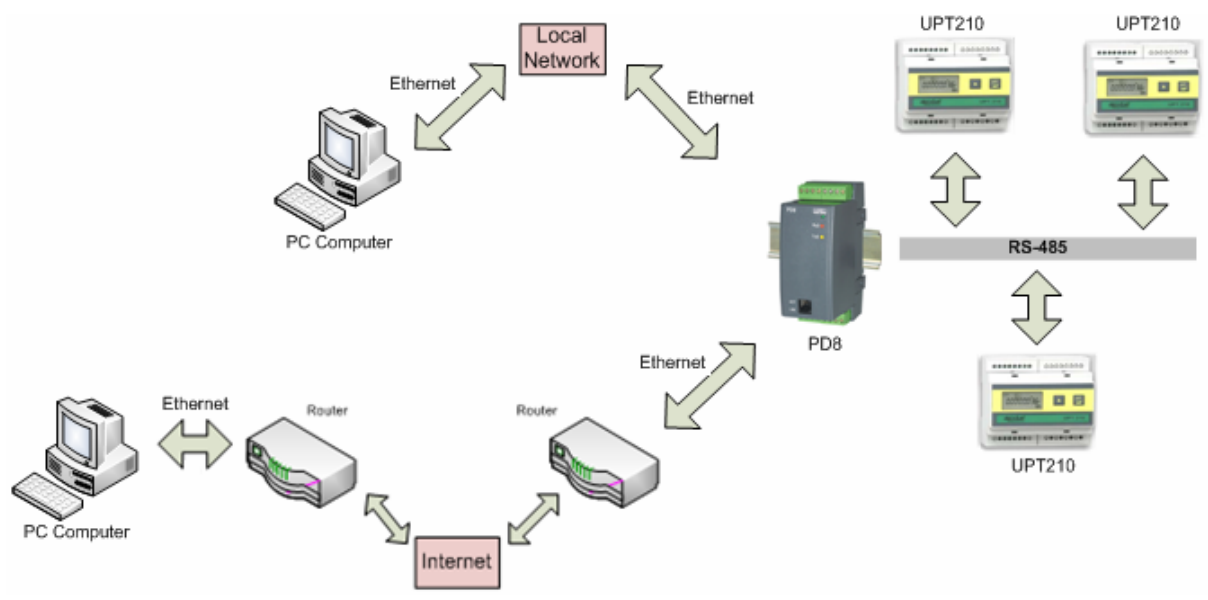

Fig. 2. System configuration using a local network or internet

\section{Monitoring Software}

The proposed monitoring software consists of two distinct applications:

- Real-time analysis

- Collect and record data

\subsection{Real-Time Analysis Application}

The developed software has the aim to make real-time analysis of all the parameters that characterize the electric network, such as voltage, current, power, active and reactive energy. The measured values can be used to build graphics load for later analysis of energy consumption in a given period of time, stored in a file, the functionality is also available in print, or simply sending the data to the mail of the person responsible for examine any issue that may exist for example.

This package can only make a connection to a single analyzer simultaneously due to the protocol limitations, which requires the reservation of a communication port on the computer for each device. On must note that this feature makes sense, because only then one can perform real-time analysis. If not the time of the n-scan counters implemented in line would suffer from such a delay that would be relevant to the energy analysis performed by this package. Fig. 3 gives an overview of the application, where a screen shot of the system acquisition is shown.

\subsection{Collect and Record Data Application}

The second application has the goal of recording all the values of active and reactive power and/or energy of each analyzer, which could be connected to the Internet or connected to a local network. In the first case, a single server is capable to collect all the data from $n$ analyzers (UPT210) connected to $m$ converters (PD8). A sampling 


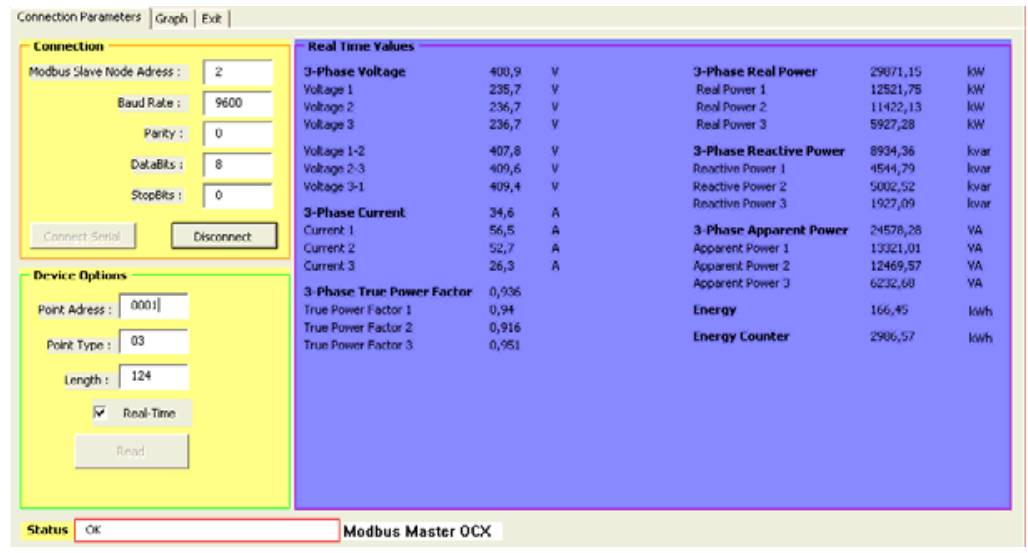

Fig. 3. Snapshot of the Real-Time implemented program

time of $10 \mathrm{msec}$ per analyzer was considered. In the second case, each local network, if there is more than one, needs a server. The application collects and records the values of energy of each analyzer considering 24 hours intervals.

If there's the need to produce a energy bill for each department associated with a specific analyzer, the server has full knowledge of what the devices contained in each network in order to be made a comparison of consumption not only from month to month but between equipments. In this perspective, it can easily detect undesired events as wasteful consumption of energy or leakage. This package has the ability to adjust the energy consumption to the rates imposed by the energy supplier. In the Portuguese case, automatic adjust to the four periods of the year as well to the different daily periods.

The previous rules were employed because, in this particular project, the system was implemented in our Institution, which is supplied by a $15 \mathrm{kV}$ medium voltage power system.

\section{Energy Consumption Analysis in Large Complexes}

This developed software package reports the consumption of a number of unlimited measurement points located in one or several complexes. Allows measuring the real costs incurred in the various sectors, departments, or even in critical equipments, with immediate benefits to users:

- Increase the transparency of energy taxation increasing the knowledge within the cost of their activities;

- Adjust the tariff policy and decide further investments in new equipment.

The user gains a powerful tool that allows benchmarking to compare the consumption of installations, equipment with other similar services, forcing those responsible for each department or sector to eliminate the observed deviations, leading to an efficient energy usage. 
This software package enables record viewing in a graphic and/or table format. It contains tools that easily allow the identification of particular power contributions and its peak value, contributions to the overall consumption of energy, and the causes of energy consumption over periods of several hours of charging.

The feature Real-Time Monitoring has some advantages that should be taken into consideration. The energy service provider usually performs monthly readings of the consumed energy or simply esteems it if the installations are small (and only charge back about 15 days later). It can run more than 6 weeks until the client is aware of its consumption and detect any abnormal behavior. To efficiently manage energy resources is important to have the knowledge of the consumption evolution in a daily and weekly basis. This is easily achieved with the developed system.

For large complexes, with several power meters, the traditional manual solution has a high cost because it requires staff to make the "journey" from meter to meter, recording the readings and entering them into a computer application for analysis. The developed system does it automatically, without the need of human intervention and with lower expenses. Fig. 4 presents the evolution of the active power for two days of consumption in the Electrical Engineering Department.

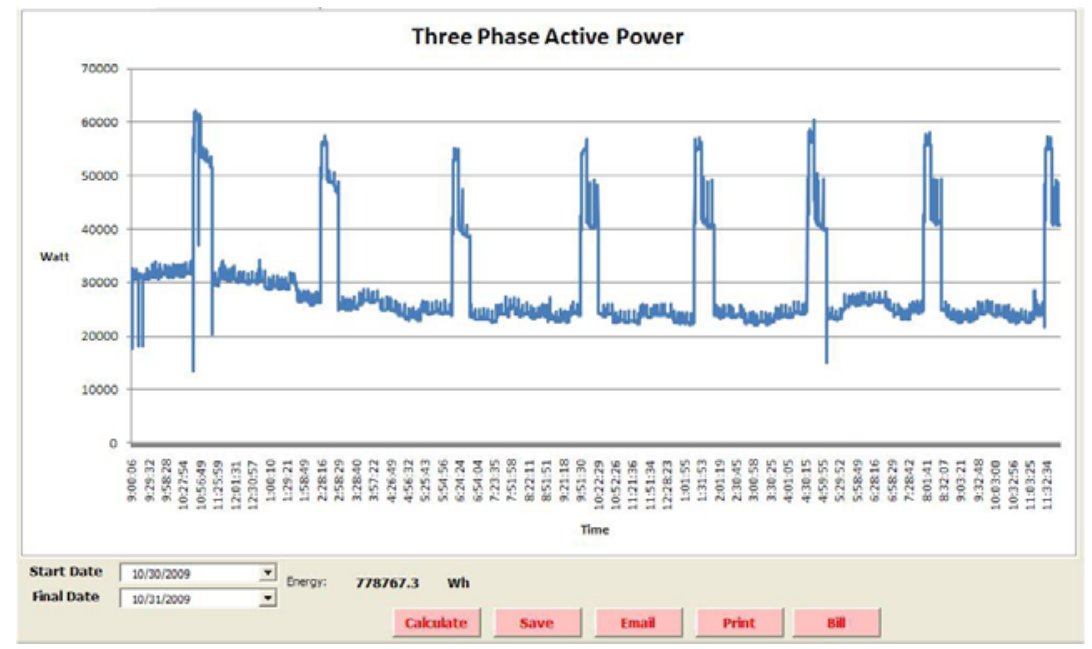

Fig. 4. Evolution of the average power

The developed software package easily adds data referring to multiple points of energy consumption. This feature makes possible to dynamically manage the consumption of a department or even a single activity, even when spread over multiple locations or buildings. The subsequent analysis of the amounts charged will help the user to optimize the consumption and pricing profile, thereby reducing the value of the energy invoice. 


\section{Conclusions}

This paper presented an energy monitoring low cost system suitable to use in large complexes, with several departments/buildings far apart. Several commercial tools are available but they become extremely expensive for large complexes with a huge amount of metering points.

The great advantage of the developed system is the use of the Modbus RTU protocol, considering the physical implementation done through Ethernet, which enables the implementation of this system in large complexes, bypassing the need to establish cabling in the whole complex area, which was practically impossible in the considered implementation.

The industrial implementation of these systems usually has the configuration RS232 to RS-485 network, stating the need of a computer in each network implemented. In this case several networks need just one computer to monitor all meters in all departments. The system also includes an analyses software package, which is very useful for energy consumption monitoring and suitable for establishing energy management policies.

The system is accessible to every industrial or commercial facility since it uses a reduced cost platform. To accomplish that, a RS-485/Ethernet converter, power meters and a standard personal computer were used. The developed monitoring system is flexible, presents a user friendly interface and a large data storage capacity.

\section{References}

1. Elhadidy, M., Shaahid, S.: Parametric Study of Hybrid (wind+solar+diesel) power generating Systems. Renew. Energy 21(2), 129-139 (2000)

2. Yeardy, M., Sweeney, J., Swan, B., Culp, C.: A Low-Cost Embedded System for Internet Based Power Measurement. International Journal of Information Technology \& Decision Making 2(4), 669-681 (2003)

3. Liao, G., Chen, Y., Lu, W., Cheng, T.: Toward Authenticating the Master in the Modbus Protocol. IEEE Transactions on Power Delivery 23(4) (2008)

4. Smith, H., Modzelewski, T.: Enhancing Energy Management Systems with Advanced RTU Capabilities. IEEE Computer Applications in Power 2, 26-29 (1989)

5. Huang, H., Yen, J., Chen, S., Ou, F.: Development of an Intelligent Energy Management Network for Building Automation. IEEE Transactions on Automation Science and Engineering 1(1) (2004)

6. Modbus-IDA, http: //www . modbus . org 